\title{
Escherichia coli Inactivation in Distilled Water Samples by Ultrasound Technology
}

\author{
M. Vidhya ${ }^{1 *}$, N. Varadharaju ${ }^{2}$, Z. John Kennedy ${ }^{2}$, D. Amirtham ${ }^{1}$ and D. Manohar Jesudas ${ }^{3}$ \\ ${ }^{1}$ Department of Food and Agricultural Process Engineering, ${ }^{2}$ Post-Harvest Technology Centre, \\ ${ }^{3}$ AMRC, AEC and RI, Tamil Nadu Agricultural University, Coimbatore-641 003, India \\ *Corresponding author
}

\section{A B S T R A C T}

Keywords

E. coli, Distilled water, Nonpasteurized, Sonicated.

Article Info

Accepted:

17 June 2017

Available Online:

10 July 2017
This work is an attempt to study the mechanism of microbial inactivation by ultrasound technology. Water used in food processing has been recently recognized as a vector for the transmitting pathogenic Escherichia coli which is a non-spore forming, gram-negative bacterium that has been implicated as the causative agent in numerous foodborne outbreaks of contaminated, non-pasteurized fruit juices. Hence, it is important to study the inactivation mechanism of any novel processes before stepping into the market. E. coli inoculated distilled water sample is subjected to ultrasound treatment at $20 \mathrm{KHz}$ frequency with single amplitude of $60 \mu \mathrm{m}$ for 15 minutes. TEM images obtained before and after sonicated samples is an evident to understand the lethality of $E$. coli bacterium by cavitation effect of ultrasound.

\section{Introduction}

An increasing consumption of fresh-like processed food products requires the development of mild processing technologies without loss of nutritional value and sensory quality of foods. Non-thermal processes have gained importance in recent years as a potential technology to replace the traditional thermal processing of foods. In recent years, several non-thermal preservation technologies have been developed in order to satisfy consumers' requirements, such as high pressure processing (HPP), ultrasound, ultraviolet (UV), pulsed electric field (PEF), irradiation, electrolyzed water and plasma technology. These innovative non- thermal techniques offer the advantages of processing at low temperatures, low energy utilization and the retention of flavors, nutrients and a fresh-like taste, while inactivating the spoilage microorganisms and enzymes. Among the aforementioned sterilization techniques, power ultrasound is an emerging technology and has a wide range of current and future applications in the food processing area.

Power ultrasound has been identified as a potential technology to meet the US Food and Drug Administration's requirement for a 5$\log$ reduction in pertinent microorganisms found in fruit juices (Salleh-Mack and Robert, 2007). The biocidal effect of ultrasound has 
been mainly attributed to physical (cavitation, mechanical effects, micro-mechanical shocks) and/or chemical (formation of free radicals due to sono-chemical reaction) principles (Butz and Tauscher, 2002; Kadkhodae and Povey, 2008; Mason et al., 1996; Pétriere et al., 2007; Piyasena et al., 2003). In liquid foods, microbial inactivation occurs due to cavitation effect and partially by formation of free radicals. When ultrasonic waves (greater than $20 \mathrm{KHz}$ frequency) passes to the medium in a continuous wave-type motion, longitudinal waves will be generated with the result that the motion creates alternative compression and refraction of the medium particles (Povey and Mason, 1998). When the negative pressure of the rarefaction cycle exceeds the attractive forces between the molecules of the liquid, a void is formed. This void or cavity in the structure takes in a small amount of vapor from the solution so that on compression it does not totally collapse, but instead continues to grow in size in successive cycles to form an acoustic cavitation bubble. There are many thousands of such bubbles in a liquid, some of which are relatively stable but some others expand further to an unstable size and undergo violent collapse to generate temperatures of about $5000 \mathrm{~K}$ and pressures of the order of $50 \mathrm{MPa}$ (Piyasena et al., 2003; Butz and Tauscher, 2002; Mason and Peters, 2002; Vollmer et al., 1998). On implosion of cavitation bubbles, results in pressure changes which are responsible for cell disruption of microbes.

There are lots of studies carried out for preserving liquid foods using ultrasound technology. Ultrasound has been applied on strawberry fruits in which decay and infection were considerably reduced along with quality maintenance (Cao et al., 2010). Knorr (2004) shows successful reduction of $E$. coli in liquid whole egg using ultrasound. Ultrasonication has proved to be one such technique (Abid et al., 2013) and is reported to retain fresh quality, nutritional value, and microbiological safety in guava juice (Cheng et al., 2007), orange juice (Valero et al., 2007), and tomato juice (Wu et al., 2008). However, we took an attempt for understanding mechanism of microbial inactivation on $E$. coli inoculated distilled water sample by ultrasound technology.

\section{Materials and Methods}

\section{Preparation of inoculum}

E. coliMTCC 433 strains collected from Microbiology Laboratory, Dept. of PHTC, Tamil Nadu Agricultural University, Coimbatore were used in the experiment. A pure culture of each strain was held at $-80^{\circ} \mathrm{C}$ in $60 \%$ glycerol until needed. Each GFPlabeled $E$. coli strain was grown overnight at $37^{\circ} \mathrm{C}$ on Luria-bertaini (Himedia, Md) plates (Sambrook et al., 1989) containing amphicillin $(100 \mu \mathrm{g} / \mathrm{ml})$. Each strain was cultivated in 1 litre of Luria-Bertani (LB) broth supplemented with amphicillin (100 $\mu \mathrm{g} / \mathrm{ml})$ at $37^{\circ} \mathrm{C}$ for $24 \mathrm{~h}$. From the freshly prepared E. coli culture, $1 \mathrm{ml}$ is taken and added to the water samples. The $E$. coli added sample was kept in rotary shaker about 20 minutes for multiplication before sonication to proceed.

\section{Ultrasound treatment}

E. coli inoculated water sample was used for sonication using an ultrasonic processor(VC 1500, Sonics and Materials Inc.,) available in the Dept. of Nano Science and Technology, TNAU, Coimbatore. $80 \mathrm{ml}$ of sample was taken in a 100-ml beaker and processed at constant frequency $(20 \mathrm{KHz})$ and single amplitude $(60 \mu \mathrm{m})$ for 15 minutes of treatment exposure. This unit consists of ultrasonic generator which converts lower frequency electrical energy into high frequency electrical energy. Piezoelectric 
transducer attached with generator helps to convert high frequency electrical energy into mechanical vibrations. Ultrasonic probe or sonotrode connected with transducer helps in transmitting mechanical vibrations into sample. Probe was submerged in the sample to a depth of $2.5 \mathrm{~cm}$ for better creation of cavitation effect.

\section{Transmission Electron Microcopy (TEM)}

Internal morphology of E. coli samples of before and after sonication were observed using Transmission Electron Microscopy (Make: FEI Technai), operated at a voltage of $100 \mathrm{KeV}$. A drop of E. coli inoculated water sample was placed on two different copper grids coated with a thin carbon film. The samples were allowed to dry at room temperature after which the copper grids were stained with a 2 wt $\%$ solution of uranyl acetate for one minute and air dried before viewing the image.

\section{Results and Discussion}

The examination of $E$. coli added sonicated water sample made with TEM proved to be a useful way of visualizing changes at the microstructure level that helps in better understanding of mechanism of microbial inactivation by ultrasound. Figures 1 and 2 show the TEM images of $E$. coli cells before and after sonication as an enlarged view. There are several kinds of damaged E. coli cells by Ultrasound. The cytoplasmic membrane appears to be retracted from the outer membrane of $E$. coli treated with ultrasound. This same result was observed by Koda et al., (2009). The cell wall of E. coli got pored and the cytoplasmic membrane content might be released in the sonicated solution by sonoporation which results in loss of structural integrity. Cavitation collapse produces alteration in disruption of the bacterial membranes. It is reasonable to speculate that a loss of integrity of the membrane by the ultrasonic treatment may lead to loss of their viability (Koda et al., 2009).

Fig.1 TEM view of E. coli colonies in untreaed sample

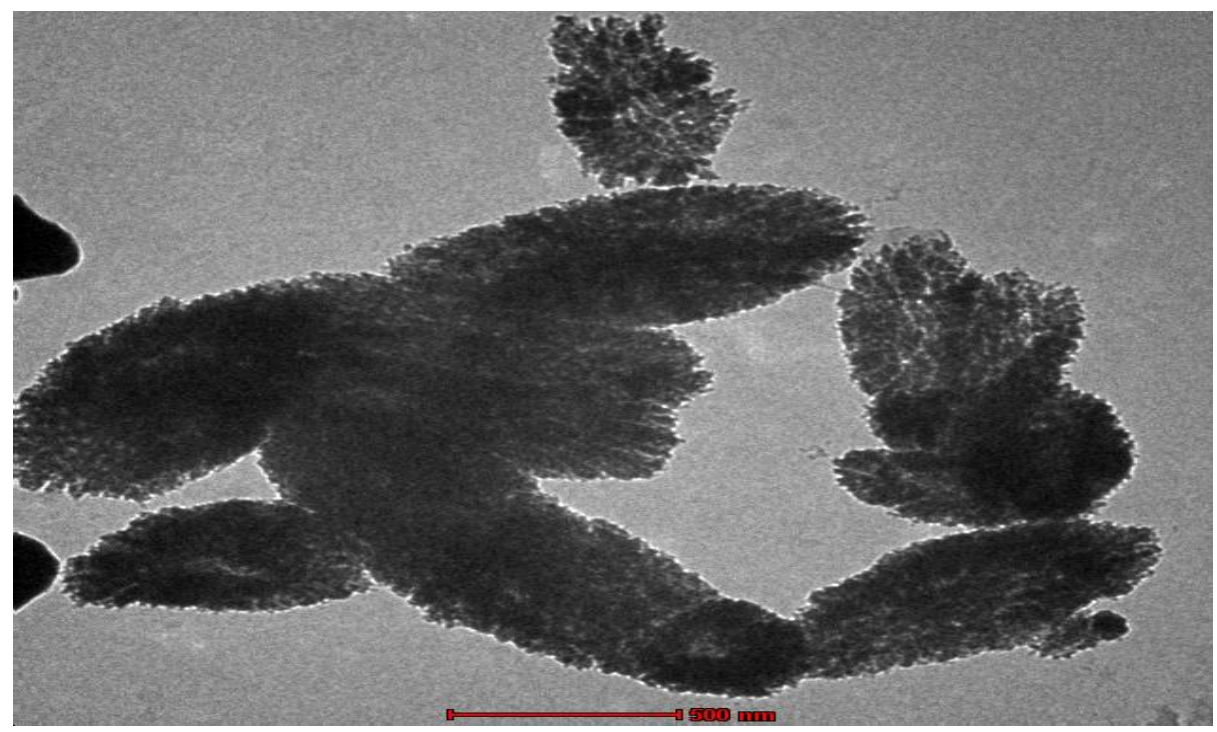


Fig.2 TEM view E. coli single cell after sonication process

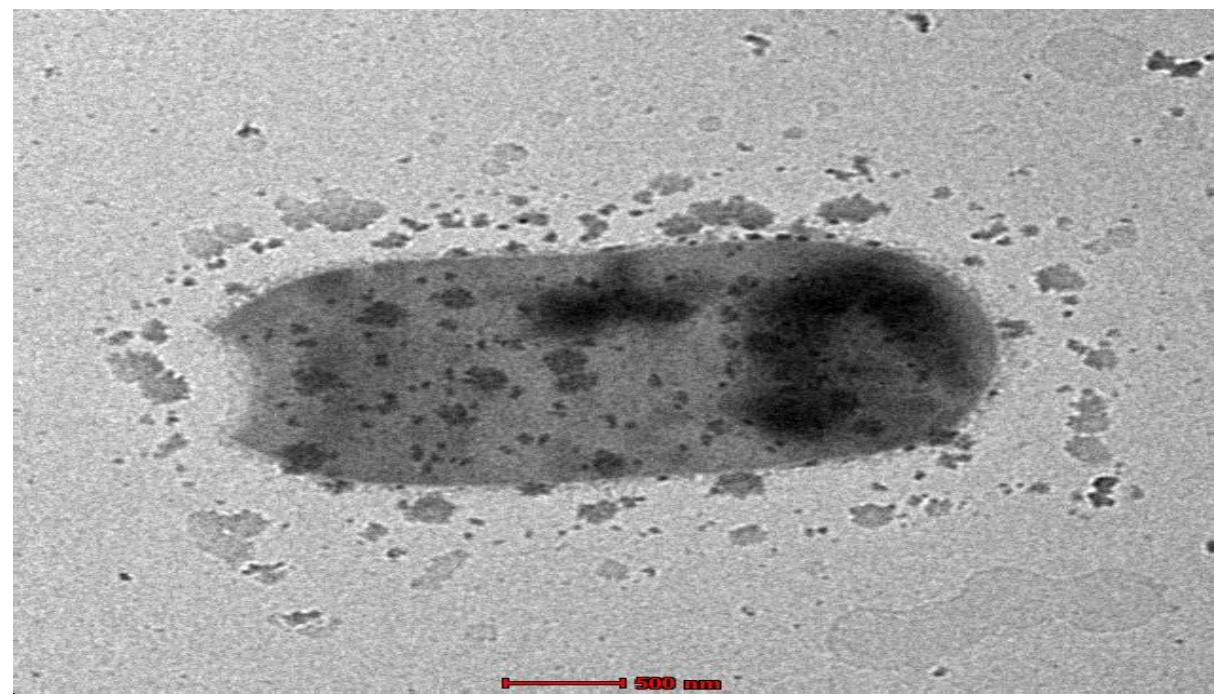

Similarly, Balasundaram and Harrison reported the TEM images of the yeast cells subjected to hydrodynamic cavitation. They observed localized damage of the yeast cell wall together with released periplasmic constitutes.

Thus, Cavitation and free radical formation are mainly responsible for $E$. coli inactivation in water samples. Cavitation results in bubble collapse with localized temperature and pressure increase damages the cell-wall of the E. coli and formation free radicals like $\mathrm{H}_{2} \mathrm{O}_{2}$ acts as a sterilizing agent which helps in preventing the multiplication of bacterial cells. Thus, ultrasound treatment, a nonthermal method definitely is a promising technology for microbial inactivation without affecting the natural quality of food stuffs.

\section{References}

Abid, M., Jabbar, S., Wu, T., Hashim, M. M., Hu, B., Lei, S., Zhang, X. 2013. Effect of ultrasound on different quality parameters of apple juice. Ultrasonics Sonochemistry, 20, 1182-1187.

Balasundaram. B and Harrison S.T.I. 2006. Disruption of Brewers' yeast by hydrodynamic cavitation: Process variables and their influence on selective release. Biotechnology Bioengineering, 94, 303

Butz, P., Tauscher, B., 2002. Emerging technologies: chemical aspects. Food Res. Int. 35, 279_284.

Cao, S., Hu, Z., Pang, B., Wang, H., Xie, H., and $\mathrm{Wu}$, F. 2010. Effect of ultrasound treatment on fruit decay and quality maintenance in strawberry after harvest. Food Control, 21, 529-532.

Cheng, L. H., Soh, C. Y., Liew, S. C., and Teh, F. F. 2007. Effects of sonication and carbonation on guava juice quality. Food Chemistry, 104, 1396-1401.

Knorr, D. (2004). Applications and potential of ultrasonics in food processing. Trends in Food Science and Technology, 15, 261-266.

Koda, S., Miyamoto, M., Toma, M., Matsuoka, T., Maebayashi, M., 2009. Inactivation of Escherichia coli and Streptococcus mutans by ultrasound at $500 \mathrm{kHz}$. Ultrasonics Sonochemistry. 16 (5), 655_659.

Kadkhodaee, R., Povey, M.J.W., 2008. Ultrasonic inactivation of Bacillus $\alpha$ amylase I effect of gas content and 
emitting face of probe. Ultrasonics Sonochemistry. 15 (2), 133_142.

Mason, T. J. (1998). Power ultrasound in food processing - the way forward. In M. J.W. Povey and T. J. Mason (Eds.), Ultrasound in Food Processing (pp.103-126). London: Blackie Academic and Professional.

Mason, T.J., Paniwnyk, L., Lorimer, J.P., 1996. The uses of ultrasound in food technology. Ultrasonics Sonochemistry. 3, 253_260.

Petrier, C., Combet, E., Mason, T., 2007. Oxygen-induced concurrent ultrasonic degradation of volatile and non-volatile aromatic compounds. Ultra-sonics Sono chemistry. 14,117_121.

Piyasena, P., Mohareb, E., McKellar, R.C., 2003. Inactivation of microbes using ultrasound: a review. Int. J. Food Microbiology. 87, 207_216.

Roberts, R.T., 1993. High intensity ultrasonics in food processing. Chem. Ind. 15,119_121

Salleh-Mack, S.Z., Roberts, J.S., 2007. Ultrasound pasteurization: the effects of temperature soluble solids organic acids and $\mathrm{pH}$ on the inactivation of
Escherichia coli ATCC 25922.

Ultrasonics Sonochemistry. 14, 323_329.

Sambrook, J., E.F. Fritch and T. Maniatis. 1989. Molecular cloning volume 2. Cold spring harbor laboratory press New York.

Suslick, K.S., 1988. Ultrasounds: Its Chemical Physical and Biological Effects. VHC Publishers, New York.

Valero, M., Recrosio, N., Saura, D., Munoz, N., Martí, N., and Lizama, V. 2007. Effects of ultrasonic treatments in orange juice processing. Journal of Food Engineering, 80, 509-516.

Vollmer, A.C., Kwakye, S., et al., 1998. Bacterial stress responses to 1megahertz pulsed ultrasound in the presence of microbubbles. Appl. Environ. Microbiol. 64 (10), 3927_3931.

Wu, J., Gamage, T. V., Vilkhu, K. S., Simons, L. K., and Mawson, R. 2008. Effect of thermo sonication on quality improvement of tomato juice. Innovative Food Science and Emerging Technologies, 9, 186-195.

\section{How to cite this article:}

Vidhya, M., N. Varadharaju, Z. John Kennedy, D. Amirtham and Manohar Jesudas, D. 2017. Escherichia coli Inactivation in Distilled Water Samples by Ultrasound Technology. Int.J.Curr.Microbiol.App.Sci. 6(7): 1182-1186. doi: https://doi.org/10.20546/ijcmas.2017.607.143 